The University of Southern Mississippi

The Aquila Digital Community

Faculty Publications

$1-1-2012$

\title{
Independent Dominating Sets In Triangle-Free Graphs
}

Wayne Goddard

Clemson University, goddard@clemson.edu

Jeremy Lyle

University of Southern Mississippi, sjlyle@olivet.edu

Follow this and additional works at: https://aquila.usm.edu/fac_pubs

Part of the Applied Mathematics Commons

\section{Recommended Citation}

Goddard, W., Lyle, J. (2012). Independent Dominating Sets In Triangle-Free Graphs. Journal of Combinatorial Optimization, 23(1), 9-20.

Available at: https://aquila.usm.edu/fac_pubs/237

This Article is brought to you for free and open access by The Aquila Digital Community. It has been accepted for inclusion in Faculty Publications by an authorized administrator of The Aquila Digital Community. For more information, please contact Joshua.Cromwell@usm.edu. 


\title{
INDEPENDENT DOMINATING SETS IN TRIANGLE-FREE GRAPHS
}

\author{
WAYNE GODDARD AND JEREMY LYLE
}

\begin{abstract}
The independent domination number of a graph is the smallest cardinality of an independent set that dominates the graph. In this paper we consider the independent domination number of triangle-free graphs. We improve several of the known bounds as a function of the order and minimum degree, thereby answering conjectures of Haviland.
\end{abstract}

\section{INTRODUCTION}

The independent domination number $i(G)$ of a simple graph $G$ is the minimum cardinality of an independent set that dominates the graph. Equivalently, it is the minimum cardinality of a maximal independent set. Recently, Haviland [2] investigated the maximum value of $i(G)$ when $G$ is triangle-free. Let $M(n, \delta)$ denote this maximum as a function of the order $n$ and the minimum degree $\delta$.

Theorem 1 (Haviland [2]).

(a) $M(n, \delta) \leq n+2 \delta-2 \sqrt{n \delta}$ if $\delta \leq(16 / 121) n$;

(b) $M(n, \delta) \leq n+3 \delta-2 \sqrt{\delta(n+3 \delta)}$ if $(16 / 121) n \leq \delta \leq(1 / 6) n$;

(c) $M(n, \delta)=n / 2$ if $(1 / 6) n \leq \delta \leq(1 / 4) n$;

(d) $n-\delta \leq M(n, \delta) \leq 3 n / 4-\delta$ if $(1 / 4) n \leq \delta \leq(1 / 3) n$;

(e) $\delta \leq M(n, \delta) \leq(2 n-\delta) / 4$ if $(1 / 3) n \leq \delta \leq(2 / 5) n$.

In this paper we:

- provide a simple proof of the value of $M(n, \delta)$ for $\delta>n / 4$;

- show that $M(n, \delta)=n / 2$ for a wider range, namely $3 n / 20 \leq \delta \leq n / 4$;

- improve slightly the upper bounds on $M(n, \delta)$ for $\delta<3 n / 20$; and

- provide a construction that shows that $M(n, \delta)>n / 2$ for $\delta<n / 10$.

The independent domination number of triangle-free graphs was also considered in $[6,3,4,1]$. The first of these considers maximal triangle-free graphs, and the remainder graphs with larger girth.

\section{Main Results}

In what follows, we will repeatedly use the fact that the neighborhood of a vertex in a triangle-free graph is independent. We start with a proof of a conjecture from [2]:

Theorem 2. (a) $M(n, \delta)=\delta$ if $n / 3 \leq \delta \leq n / 2$;

(b) $M(n, \delta)=n-2 \delta$ if $n / 4 \leq \delta \leq n / 3$.

Proof. Let $G$ be a triangle-free graph. If $G$ has diameter 2, then the neighborhood of any vertex dominates the graph by the diameter condition; so $i(G) \leq \delta$. If $G$ has diameter 3 or more (or is disconnected), then there are two non-adjacent vertices $v_{1}$ and $v_{2}$ with disjoint neighborhoods. Any maximal independent set containing these two vertices has cardinality at most $n-2 \delta$. Therefore,

$$
i(G) \leq \max \{\delta, n-2 \delta\},
$$

which implies that the values in the theorem are upper bounds for $M(n, \delta)$. 
The fact that this bound is best possible was shown by Haviland [2] (and stated in Theorem 1). For $\delta \geq n / 3$, take $K(\delta, n-\delta)$. For $n / 4 \leq \delta \leq n / 3$, start with $K_{4}$ and subdivide two edges; then expand each of the original four vertices to $\delta / 2$ vertices, and both the subdivision vertices to $n / 2-\delta$ vertices.

We next provide a simple generalization of the above argument. While this immediately gives a simple proof of the upper bounds from Theorem 1, the main purpose is that we will thereafter refine this argument to give us better results for $\delta<n / 6$. Recall that a packing in a graph is a set of vertices that are mutually at distance at least 3 .

Theorem 3. Let $f(n, \delta)$ be the piecewise linear function defined for $0<\delta \leq n / 2$ by

$$
f(n, \delta)=\frac{k-1}{k} n-(k-2) \delta \text { for } \frac{1}{k(k+1)} n \leq \delta \leq \frac{1}{k(k-1)} n \text { and } k=2,3, \ldots
$$

Then $M(n, \delta) \leq f(n, \delta)$.

Proof. Let $G$ be a triangle-free graph and let $P$ be any maximal packing of $G$. Say $P=\left\{v_{1}, \ldots, v_{k}\right\}$. For each $1 \leq i \leq k$, form a maximal independent set $I_{i}$ by starting with $N\left(v_{i}\right) \cup\left(P-\left\{v_{i}\right\}\right)$. Let $N=\bigcup_{i} N\left(v_{i}\right)$. By the definition of a packing, the $N\left(v_{i}\right)$ are disjoint and so $|N| \geq k \delta$. By the maximality of $P, N$ dominates $G$. Therefore, every vertex outside $N$ appears in at most $k-1$ of the $I_{i}$. It follows that

$$
k i(G) \leq(k-1)(n-|N|)+|N| \leq(k-1) n-(k-2) k \delta,
$$

and thus $i(G) \leq((k-1) / k) n-(k-2) \delta$.

Note that the expression gives $\delta$ for $k=1$ and $n / 2$ for $k=2$. It follows that

$$
M(n, \delta) \leq \max _{k \geq 2} \frac{k-1}{k} n-(k-2) \delta .
$$

By straight-forward algebra, the bound of the theorem follows.

For example, this theorem reproves that $M(n, \delta) \leq n / 2$ for $\delta \geq n / 6$. And by a little algebra, it can be shown that for $\delta<n / 6$, this bound is strictly better than Theorem 1 except at points of the form $\delta=n / k^{2}$ (where they match). But we will immediately improve this averaging argument by considering the properties of triangle-free graphs that would not satisfy an improved version of the upper bound. In particular we will prove the following result:

Theorem 4. For any triangle-free graph $G$ with $i(G) \geq n / 2$,

$$
i(G) \leq \max _{k \geq 3} \frac{k-1}{k} n-(k-2) \delta-\frac{1}{3 k} \delta .
$$

We defer the proof to Section 4. Using different methods, we will prove in Section 5 another bound:

Theorem 5. For any triangle-free graph $G$ with $i(G) \geq n / 2$,

$$
i(G) \leq n+\frac{22}{5} \delta-\frac{2 \sqrt{30}}{5} \sqrt{\delta(n+4 \delta)} .
$$

From Theorems 4 and 5, straight-forward algebraic manipulation proves:

Theorem 6. (a) $M(n, \delta)=n / 2$ for $\delta \geq(3 / 20) n$. 


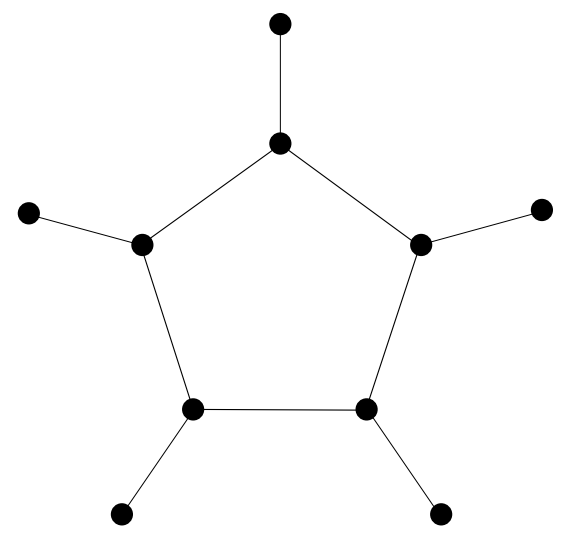

FiguRE 1. A graph whose blow-up has $i(G)>n / 2$

(b)

$$
M(n, \delta) \leq \begin{cases}(2 / 3) n-(10 / 9) \delta & \text { if }(3 / 35) n \leq \delta \leq(3 / 20) n \\ (3 / 4) n-(25 / 12) \delta & \text { if }(3 / 59) n \leq \delta \leq(3 / 35) n \\ (4 / 5) n-(46 / 15) \delta & \text { if }(3 / 89) n \leq \delta \leq(3 / 59) n \\ (5 / 6) n-(73 / 18) \delta & \text { if }(3 / 125) n \leq \delta \leq(3 / 89) n \\ (6 / 7) n-(106 / 21) \delta & \text { if }(3 / 167) n \leq \delta \leq(3 / 125) n \\ (7 / 8) n-(145 / 24) \delta & \text { if }(3 / 215) n \leq \delta \leq(3 / 167) n \\ (8 / 9) n-(190 / 27) \delta & \text { if }(3 / 269) n \leq \delta \leq(3 / 215) n \\ (9 / 10) n-(241 / 30) \delta & \text { if }(3 / 329) n \leq \delta \leq(3 / 269) n \\ (10 / 11) n-(298 / 33) \delta & \text { if }((8025+495 \sqrt{11}) / 1096984) n \leq \delta \leq(3 / 329) n \\ n+\frac{22}{5} \delta-\frac{2 \sqrt{30}}{5} \sqrt{\delta(n+4 \delta)} & \text { if } \delta \leq((8025+495 \sqrt{11}) / 1096984) n\end{cases}
$$

\section{Triangle-free Graphs with No Small Maximal Independent Sets}

Haviland [2] was unable to find examples of triangle-free graphs with $i(G)>n / 2$. This we do, by constructing graphs with $i(G)>n / 2$ for all $0<\delta(G)<n / 10$.

Let $a, b$ be positive integers with $a \leq b$ and let $H$ be a triangle-free graph. We define a trianglefree graph $H^{\prime}(a, b)$ as follows. Let $H^{\prime}$ be the corona of $H$; that is, for each vertex in $H$ add a new end-vertex adjacent to only that vertex. Then form $H^{\prime}(a, b)$ by expanding each original vertex of $H$ to $a$ vertices and each end-vertex to $b$ vertices.

Lemma 1. If $H$ has order $m$, then $H^{\prime}(a, b)$ has order $m(a+b)$, minimum degree $a$, and $i\left(H^{\prime}(a, b)\right)=$ $m b+\alpha(H) \cdot(a-b)$, where $\alpha(H)$ denote the size of the largest independent set of $H$.

Proof. The statement about the order and minimum degree is obvious. So consider the value of $i\left(H^{\prime}(a, b)\right)$.

As is true for all graphs, if we take one vertex from a pair of independent vertices with the same neighborhood for an independent dominating set, then we must take the other. It follows that for each original vertex $v$ of $H$, we must take either all $a$ vertices of the expansion of $v$ or all $b$ vertices of the expansion of $v$ 's coronal end-vertex. Since $b \geq a$, we must take as many original vertices as possible; that is, to minimize the size of the independent dominating set, we must begin with a maximum independent set of $H$.

For example, if $H=K_{2}$, then $H^{\prime}=P_{4}$ and $H^{\prime}(a, n / 4-a)$ is a triangle-free graphs with $i(G)=n / 2$ for all $\delta(G)=a$ from 1 up to $n / 4$, as observed by Haviland. However, if we take $H=C_{5}$, then $i\left(C_{5}^{\prime}(a, n / 5-a)\right)$ exceeds $n / 2$ for all $\delta=a<n / 10$. 


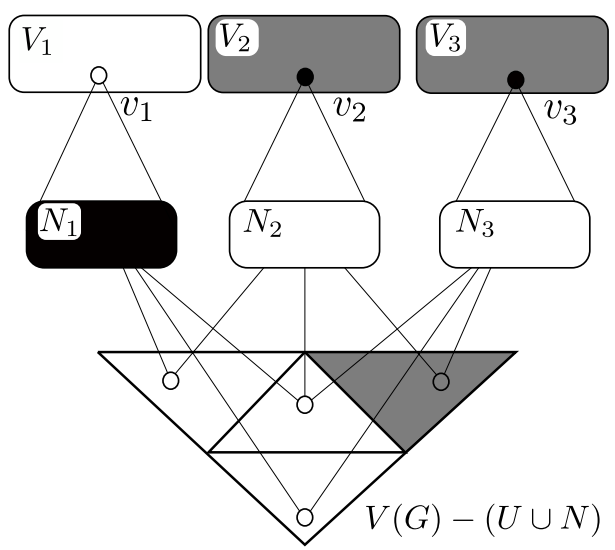

FiguRE 2. An illustration of the improved partition for the case $k=3$. Regions/vertices shaded black are contained in $I_{1}$, regions shaded gray may contain vertices in $I_{1}$, and unshaded regions contain no vertices in $I_{1}$.

Indeed, one can construct triangle-free graphs with $i(G)=n-o(n)$. Let $G_{t}$ denote a Ramsey graph for $R(3, t)$, and $m_{t}$ denote the number of vertices. Kim [5] showed that $m_{t}$ is $\Theta\left(t^{2} \log t\right)$. If we choose $t=\sqrt[3]{n / 2 a}$, then by Lemma 1 , we obtain graphs $G_{t}^{\prime}\left(a, n / m_{t}-a\right)$ with independence number $n-O\left(\sqrt[3]{n^{2} \delta}\right)$.

\section{Proof of Theorem 4}

Define $f_{2}(n, \delta)=n / 2$, and for $k \geq 3$, define

$$
f_{k}(n, \delta)=\frac{k-1}{k} n-(k-2) \delta-\frac{1}{3 k} \delta .
$$

The goal is to prove that $M(n, \delta) \leq \max _{k} f_{k}(n, \delta)$. This is an immediate consequence of the following lemma:

Lemma 2. Let $G$ be a triangle-free graph. Let $P$ be any maximal packing of $G$ containing a vertex $v_{1}$ of maximum degree $\Delta$, and let $|P|=k$. If $k=2$ then $i(G) \leq n / 2$; and if $k \geq 3$ then

$$
i(G) \leq \max \left\{f_{k}(n, \delta), f_{k-1}(n, \delta)\right\} .
$$

To prove this lemma, we need to build on the use of packings as given in the proof of Theorem 3 . By that proof we already know the result for $k=2$. So we can assume $k \geq 3$.

Let $P=\left\{v_{1}, \ldots, v_{k}\right\}$, so that $v_{1}$ is a vertex of maximum degree. Recall that we created independent dominating sets $I_{1}, \ldots, I_{k}$. We defined $N=\bigcup N\left(v_{i}\right)$. Let $U$ be those vertices outside $N$ that are adjacent to exactly one of the $N\left(v_{i}\right)$; note that $U \supseteq P$. Then it follows that every vertex outside $U \cup N$ is in at most $k-2$ of the $I_{i}$, and thus

$$
k \cdot i(G) \leq|N|+(k-1)|U|+(k-2)(n-|N \cup U|)
$$

so that

$$
i(G) \leq \frac{k-2}{k} n+\frac{|U|}{k}-\frac{k-3}{k}|N| .
$$

To simplify the equations, we will write $N_{i}=N\left(v_{i}\right)$. Define a partition of $U$ by

$$
U_{i}=\left\{u \in V(G): N(u) \cap N_{i} \neq \emptyset, N(u) \cap N_{j}=\emptyset(j \neq i)\right\} .
$$

This set-up is illustrated in Figure 2.

Claim 1. Suppose $G$ has a packing as previously described, with $k \geq 3$ vertices, so that $i(G)>$ $\max \left\{f_{k}(n, \delta), f_{k-1}(n, \delta)\right\}$. We may assume: 
(a) $\Delta<(4 / 3) \delta$.

(b) There are $U_{i}, U_{j},(i \neq j)$ with $\left|U_{i}\right|,\left|U_{j}\right|>n / k-\left(3 k^{2}-2 k+1\right) \delta /\left(3 k^{2}-3 k\right)$.

(c) For all $i$, and any $u \in U_{i},\left|N(u) \cap\left(V(G)-N_{i}\right)\right|<(1 / 3) \delta$.

(d) For all $i,\left|U_{i}\right|>\left|N_{i}\right|$.

(e) For all $i$, there is a vertex $w_{i} \in N_{i}$ such that $\left|N\left(w_{i}\right) \cap U_{i}\right|>((2 / 3) \delta)\left|U_{i}\right| /\left|N_{i}\right|$.

Proof. (a) Since $i(G)>f_{k}(n, \delta)$ and $|N| \geq k \delta$, it follows from Equation 2 that $|U|>n-(k+(1 / 3)) \delta$. Since $U$ and $N$ are disjoint, it follows that the degree of $v_{1}$ is at most $n-(k-1) \delta-|U|<(4 / 3) \delta$.

(b) There must be at least one set $U_{i}$ such that $\left|U_{i}\right| \geq|U| / k$. Suppose all other sets contain at most $|U| / k-2 \delta /(3 k(k-1))$ vertices. Then, $\left|U_{i}\right|>|U| / k+2 \delta /(3 k)$. In part (a) we showed that $|U|>n-(k+(1 / 3)) \delta$. Consider $I_{i}$. Then one obtains

$$
\begin{aligned}
i(G) & \leq n-\left(|N|-\left|N_{i}\right|\right)-\left|U_{i}\right| \\
& \leq n-(k-1) \delta-\left(\frac{n-(k+(1 / 3)) \delta}{k}+\frac{2 \delta}{3 k}\right) \\
& =f_{k}(n, \delta) .
\end{aligned}
$$

Hence there is some $U_{j}$ with more than $|U| / k-2 \delta /(3 k(k-1))$ vertices, and the result follows.

(c) Suppose $u \in U_{i}$ and $\left|N(u) \cap\left(V(G)-N_{i}\right)\right| \geq(1 / 3) \delta$. Note that for $\ell \neq i, N(u) \cap N_{\ell}=\emptyset$, by the definition of $U_{i}$. Then $N(u) \cap\left(V(G)-N_{i}\right)$ can be partitioned into the two sets, $S_{1}=N(u) \cap U$ and $S_{2}=N(u) \cap(V(G)-(U \cup N))$. Also, $N(u) \cap\left(P-\left\{v_{\ell}\right\} \cup N_{\ell}\right)=\emptyset$; so when we construct the sets $I_{\ell}$, we can specify that they all contain $u$. Then, any vertex in $S_{1} \cup S_{2}$ is contained in $I_{\ell}$ (except $\ell=i$ ). Therefore, refine Equation 1 by considering the vertices in $S_{1} \cup S_{2}$ separately:

$$
\begin{aligned}
k \cdot i(G) & \leq|N|+(k-1)\left(|U|-\left|S_{1}\right|\right)+(k-2)\left(n-|N \cup U|-\left|S_{2}\right|\right)+\left|S_{1} \cup S_{2}\right| \\
& =(k-2) n+|U|-(k-3)|N|-(k-2)\left|S_{1}\right|-(k-3)\left|S_{2}\right| .
\end{aligned}
$$

Use the bounds $|U| \leq\left|V(G)-\left(N \cup S_{2}\right)\right|=n-|N|-\left|S_{2}\right|$, and $|N| \geq k \delta$, to obtain

$$
k \cdot i(G) \leq(k-1) n-(k-2) k \delta-(k-2)\left|S_{1} \cup S_{2}\right|
$$

so that

$$
i(G) \leq \frac{(k-1)}{k} n-(k-2) \delta-\frac{(k-2)}{3 k} \delta \leq f_{k}(n, \delta) .
$$

(d) Consider the $k-1$ independent dominating sets $I_{j}$ such that $j \neq i$. Then, vertices of $N_{i}$ appear no times; vertices of $N-N_{i}$ appear once; vertices of $V(G)-\left(N \cup U_{i}\right)$ appear at most $k-2$ times; and vertices of $U_{i}$ might appear in each $I_{j}$. Thus

$$
\begin{aligned}
(k-1) i(G) & \leq|N|-\left|N_{i}\right|+(k-2)(n-|N|)+\left|U_{i}\right| \\
& =(k-2) n-(k-3)|N|+\left(\left|U_{i}\right|-\left|N_{i}\right|\right)
\end{aligned}
$$

Substitute $|N| \geq k \delta$ to obtain

$$
\begin{aligned}
i(G) & \leq \frac{k-2}{k-1} n-(k-3) \delta-\frac{k-3}{k-1} \delta+\frac{1}{k-1}\left(\left|U_{i}\right|-\left|N_{i}\right|\right) \\
& \leq f_{k-1}(n, \delta)+\frac{1}{k-1}\left(\left|U_{i}\right|-\left|N_{i}\right|\right) .
\end{aligned}
$$

Therefore, we may assume $\left|U_{i}\right|-\left|N_{i}\right|>0$.

(e) Let $Z$ be the number of edges between $N_{i}$ and $U_{i}$. Since each vertex $u \in U_{i}$ has less than $(1 / 3) \delta$ neighbors in $V(G)-N_{i}$ (by part (c)),

$$
\sum_{u \in N_{i}}\left|N(u) \cap U_{i}\right|=Z>((2 / 3) \delta)\left|U_{i}\right|
$$


Taking the average, this implies that there is a suitable vertex $w_{i} \in N_{i}$.

This completes the proof of Claim 1.

Proof of Lemma 2. Let $w_{1} \in N_{1}$ be a vertex satisfying part (e) of Claim 1 for $i=1$. By part (d) of that claim, this implies that $\left|N\left(w_{1}\right) \cap U_{1}\right|>(2 / 3) \delta$. By part (a) of that claim, $w_{1}$ has degree less than $(4 / 3) \delta$, which implies $\left|N\left(w_{1}\right) \cap N\right|<(4 / 3) \delta-(2 / 3) \delta=(2 / 3) \delta$.

Define $N_{j}^{\prime}=N_{j}-N\left(w_{1}\right)$ for $j \geq 2$. Note that $\left|N_{j}^{\prime}\right|>\delta-(2 / 3) \delta=(1 / 3) \delta$. So, by part (c) of Claim 1, any vertex $v \in U_{j}$ is adjacent to some vertex in $N_{j}^{\prime}$. Therefore, each set $N_{j}^{\prime}$ dominates $U_{j}$.

At this point, we note that $w_{1}$ cannot dominate $U_{1}$, since by part (c) of the above claim, $\left|U_{1}\right|>$ $\left|N_{1}\right|=\Delta$. Therefore, let $w_{1}^{\prime}$ be an undominated vertex in $U_{1}$. Consider a maximal independent set $I_{j}^{\prime}$ containing $\left\{w_{1}, w_{1}^{\prime}\right\} \cup N_{j}^{\prime} \cup\left\{v_{\ell}\right\}_{\ell \neq 1, j}$. Then, this set takes no element from $N$ except those in $N_{j}$ and $N_{1}-N\left(w_{1}^{\prime}\right)$. Also, no element from $U_{j}$ is taken, and no element from $N\left(w_{1}\right) \cap U_{1}$. Therefore,

$$
\left|I_{j}^{\prime}\right| \leq n-|N|+\left|N_{j}\right|+\left|N_{1}\right|-\left|U_{j}\right|-(4 / 3) \delta
$$

Choose $j$ to satisfy part (b) of Claim 1 . Note that $|N|-\left|N_{j}\right|-\left|N_{1}\right|>(k-2) \delta$. Thus

$$
\begin{aligned}
i(G) & \leq n-(k-2) \delta-\left(\frac{n-(k+(1 / 3)) \delta}{k}-\frac{2 \delta}{3 k(k-1)}\right)-(4 / 3) \delta \\
& \leq \frac{k-1}{k} n-(k-2) \delta-\frac{1}{3} \delta\left(1-\frac{1}{k}-\frac{2}{k(k-1)}\right) .
\end{aligned}
$$

Since $1 / k \leq 1-1 / k-2 /\left(k^{2}-k\right)$ for $k \geq 3$, we obtain

$$
i(G) \leq \frac{k-1}{k} n-(k-2) \delta-\frac{1}{3 k} \delta=f_{k}(n, \delta) .
$$

This completes the proof of Lemma 2.

At this point we note that one can prove a more general version of Claim 1 , with $\varepsilon_{k}$ replacing $1 / 3$ in the definition of $f_{k}(n, \delta)$ in the following way:

$$
f_{k}\left(n, \delta, \varepsilon_{k}\right)=\frac{k-1}{k} n-(k-2) \delta-\frac{\varepsilon_{k}}{k} \delta, \quad \varepsilon_{k}<1 .
$$

One can then improve the bounds slightly, in particular for some $\varepsilon_{k}>1 / 3$ for $k \geq 6$, by applying a different argument to replace the proof of Lemma 2. However, substantial improvements beyond these would seem to require a new attack.

\section{Proof of Theorem 5}

In this section, we consider an alternative approach to bound $i(G)$. The motivation for this approach comes from examining the graphs for which $i(G)>n / 2$. To force $i(G)>n / 2$, the graph $G$ must paradoxically contain many subgraphs (odd cycles) containing only small maximal independent sets.

We partition the vertices of $G$ into sets $\mathcal{A}, \mathcal{B}$, and $\mathcal{C}$ as follows. Let $\Gamma$ be a maximal collection of vertex-disjoint odd induced cycles in $G$. (Such a collection can, for example, be constructed by repeatedly removing the shortest odd cycle remaining until there is no odd cycle left.) Let $\mathcal{C}$ be the set of vertices in $\Gamma$ and $t$ the number of cycles in $\Gamma$. Then the graph $G-\mathcal{C}$ is bipartite. Let $\mathcal{A}$ be the isolated vertices of $G-\mathcal{C}$ (that is, $\mathcal{A}=\{v \in V(G)-\mathcal{C}: N(v) \cap(V(G)-\mathcal{C})=\emptyset\}$ ) and let $\mathcal{B}$ be the non-isolated vertices in $G-\mathcal{C}$.

Lemma 3 (Bound A).

$$
i(G) \leq \frac{1}{2}(n+|\mathcal{A}|-t) .
$$


Proof. The vertices of $\mathcal{B}$ can be dominated by $|\mathcal{B}| / 2$ vertices, since the graph induced by $\mathcal{B}$ is bipartite with no isolates. Extend this to an independent dominating set $D$ of $G$. Since the graph induced by $\mathcal{C}$ is spanned by $t$ disjoint odd cycles, it follows that $D$ contains at most $(|\mathcal{C}|-t) / 2$ vertices of $\mathcal{C}$. Thus,

$$
i(G) \leq|\mathcal{B}| / 2+|\mathcal{A}|+(|\mathcal{C}|-t) / 2=(n+|\mathcal{A}|-t) / 2 .
$$

Another way to construct a bound is to find a cycle adjacent to many vertices in $\mathcal{A}$, and start by choosing a subset from this cycle to dominate many vertices in $\mathcal{A}$. For this:

- Choose the $\Gamma$ for which the number $t$ of cycles is maximized. And subject to this, choose $\Gamma$ that minimizes $|\mathcal{A}|$.

Lemma 4 (Bound B). For the choice of $\Gamma$,

$$
i(G) \leq \frac{1}{2}(n+|\mathcal{A}|+|\mathcal{B}|-t)-\frac{2 \delta}{5 t}|\mathcal{A}| .
$$

Proof. We start by observing some structure on the edges between $\mathcal{A}$ and $\mathcal{C}$.

Claim 2. Let $C \in \Gamma$ and $y \in \mathcal{A}$. If $y$ has at least two neighbors in $C$, then

(i) y has exactly two neighbors in $C$ and these two neighbors have a common neighbor $v$ on $C$; and (ii) $v$ has no neighbor in $\mathcal{A}$.

Proof. (i) Say $y$ has $r$ neighbors on $C$. If we were to remove these $r$ vertices from $C$, it would leave a collection of $r$ paths. Since $C$ has an odd number of vertices, at least one of these paths, say $P$, has an even number of vertices. Say $P$ is bracketed by neighbors $u$ and $w$ of $y$.

Then one can replace $C$ in $\Gamma$ by the odd induced cycle containing $y, u, w$, and $P$. The unused portion $C-(P \cup\{u, w\})$ either creates an odd cycle with $(\mathcal{A}-y) \cup \mathcal{B}$, which contradicts the maximality of $t$ in our choice of $\Gamma$, or if longer than one vertex, becomes part of $\mathcal{B}$, which contradicts the minimality of $\mathcal{A}$ in our choice. Thus the only possibility is that $P$ is one vertex, say $v$. That is, $u$ and $w$ are exactly distance two apart. And therefore they are the only neighbors of $y$ on $C$.

(ii) Suppose $v$ has a neighbor $x \in \mathcal{A}$. Then one can replace $C$ in $\Gamma$ by the odd induced cycle $C-\{v\}+\{y\}$. If $v$ is in an odd cycle with $(\mathcal{A}-y) \cup \mathcal{B}$, then this contradicts the maximality of $t$; and if not, this contradicts the minimality of $\mathcal{A}$, as above.

Claim 3. Let $C \in \Gamma$. Then there is an independent set $I \subseteq V(C)$ such that

$$
|N(I) \cap \mathcal{A}| \geq 2 M_{C} / 5,
$$

where $M_{C}$ is the number of edges from $\mathcal{A}$ to $C$.

Proof. Define a vertex of $C$ as insular if its two neighbors on $C$ have a common neighbor in $\mathcal{A}$. By (ii) above, insular vertices have no neighbors in $\mathcal{A}$ and cannot be consecutive on $C$. Let $v_{1}, v_{2}, \ldots, v_{\ell}, v_{1}$ be the non-insular vertices on $C$ in order. It follows that $\ell \geq 3$. There are three cases.

(1) $\ell$ is even. Then partition the non-insular vertices into sets $I_{1}=\left\{v_{1}, v_{3}, \ldots, v_{\ell-1}\right\}$ and $I_{2}=\left\{v_{2}, v_{4}, \ldots, v_{\ell}\right\}$. Clearly, both $I_{1}$ and $I_{2}$ are independent sets.

By (i) above, if a vertex $y \in \mathcal{A}$ has two neighbors on $C$, these neighbors are consecutive considering only the insular vertices on $C$. Thus $y$ is adjacent to both $I_{1}$ and $I_{2}$. It follows that $\left|N\left(I_{1}\right) \cap \mathcal{A}\right|+\left|N\left(I_{2}\right) \cap \mathcal{A}\right|=M_{C}$. So for some $j$ we have $\left|N\left(I_{j}\right) \cap \mathcal{A}\right| \geq M_{C} / 2$.

(2) $\ell$ is odd and $\ell \geq 5$. Then WLOG $v_{1}$ is the non-insular vertex with the fewest neighbors in $\mathcal{A}$. Then $|N(x) \cap \mathcal{A}| \leq M_{C} / \ell$. Define independent sets $I_{1}=\left\{v_{3}, v_{5}, \ldots, v_{\ell}\right\}$ and $I_{2}=$ $\left\{v_{2}, v_{4}, \ldots, v_{\ell-1}\right\}$.

As before, if a vertex $y \in \mathcal{A}$ has two neighbors in $C$, then they are not in the same $I_{j}$. Thus $\left|N\left(I_{1}\right) \cap \mathcal{A}\right|+\left|N\left(I_{2}\right) \cap \mathcal{A}\right|=M_{C}-\left|N\left(v_{1}\right) \cap \mathcal{A}\right|$, and so for some $j$ we have $\left|N\left(I_{j}\right) \cap \mathcal{A}\right| \geq$ $\left(M_{C}-M_{C} / \ell\right) / 2 \geq 2 M_{C} / 5$. 
(3) $\ell=3$. If this case, $C$ must be a 5-cycle. WLOG $w_{2}$ and $w_{3}$ are adjacent. Then let $I_{1}=\left\{w_{1}, w_{2}\right\}$ and $I_{2}=\left\{w_{1}, w_{3}\right\}$. It follows that if a vertex $y \in \mathcal{A}$ has two neighbors on $C$, then one of these must be $w_{1}$, and so $y \in N\left(I_{1}\right) \cap N\left(I_{2}\right)$. Thus $\left|N\left(I_{1}\right) \cap \mathcal{A}\right|+\left|N\left(I_{2}\right) \cap \mathcal{A}\right|=M_{C}$, and conclude as before.

Now to finish the proof of Lemma 4 . Let $C^{*}$ be the cycle of $\Gamma$ with the maximum number $M$ of edges between it and $\mathcal{A}$. Since every neighbor of every vertex on $\mathcal{A}$ is in $\mathcal{C}$, and there are $t$ cycles in $\Gamma$, it follows that $M \geq|\mathcal{A}| \delta / t$.

Let $I$ be the independent subset of $V\left(C^{*}\right)$ guaranteed by the above claim. Extend this to an independent dominating set $D$ of $G$. By the claim, $I$ dominates at least a $(2 \delta) /(5 t)$ fraction of $\mathcal{A}$. And as before, $D$ contains at most $(|\mathcal{C}|-t) / 2$ vertices of $\mathcal{C}$. Thus,

$$
i(G) \leq|\mathcal{A}|-\frac{2 \delta}{5 t}|\mathcal{A}|+|\mathcal{B}|+\frac{1}{2}(|\mathcal{C}|-t)=\frac{1}{2}(n+|\mathcal{A}|+|\mathcal{B}|-t)-\frac{2 \delta}{5 t}|\mathcal{A}|,
$$

as required.

We now combine Bounds A and B using algebra and calculus to obtain an upper bound on $i(G)$ that does not depend on the partition $\mathcal{A}, \mathcal{B}$, and $\mathcal{C}$.

Proof of Theorem 5. Since every cycle in $\Gamma$ has length at least 5 , it holds that $n \geq|\mathcal{A}|+|\mathcal{B}|+5 t$. Since Bound B is increasing in $|\mathcal{B}|$ (and Bound A does not depend on $|\mathcal{B}|$ ), it follows that we may assume $|\mathcal{B}|$ is its maximum value, namely $n-|\mathcal{A}|-5 t$.

Thus, letting $a=|\mathcal{A}|$,

$$
i(G) \leq \min \left\{\begin{array}{l}
(n+a-t) / 2 \\
n-3 t-2 \delta a /(5 t) .
\end{array}\right.
$$

We need to maximize this minimum as a function of $a$ and $t$.

For a fixed value of $t$, both expressions are linear in $a$, one increasing and one decreasing. It follows that the minimum is maximized at the value of $a$ where these two expressions are equal, namely $5(n-5 t) t /(5 t+4 \delta)$. Thus,

$$
i(G) \leq \max _{t} \frac{2 \delta(n-t)+5 t(n-3 t)}{4 \delta+5 t} .
$$

By calculus, we find the maximum value occurs at

$$
t^{*}=\frac{\sqrt{30} \sqrt{\delta n+4 \delta^{2}}-12 \delta}{15} .
$$

Substituting this value in gives the desired bound:

$$
i(G) \leq n+\frac{22}{5} \delta-\frac{2 \sqrt{30}}{5} \sqrt{\delta(n+4 \delta)} .
$$

One benefit of this approach is that it can be extended to give improved bounds on $i(G)$ when $G$ has odd girth $g$ by using $|\mathcal{C}| \geq g t$ and improving Claim 3 .

\section{Conclusion}

We suspect that the construction given in Section 3 gives the triangle-free graphs with the largest minimum independent dominating sets for given $n$ and small $\delta$. In view of this relationship between $i(G)$ and $R(3, t)$, it seems a much more difficult problem to determine sharp bounds on $i(G)$ for triangle-free graphs than for general graphs. An appropriate intermediate step might be the following question.

Question 1. Is is true that any triangle-free graph $G$ with $\delta \geq n / 10$ has $i(G) \leq n / 2$. 
If true, this bound is sharp by the corona of a 5-cycle, shown in Figure 1.

Graphs with large girth $(\geq 5)$ have the benefit that independent neighborhoods extend to independent sets at distance $k$, and there is very little local interaction between two vertices. This enables very good constructive and probabilistic bounds on $i(G)$, addressed in $[3,4,1]$. However, if small even cycles are not allowed, the minimum degree for such graphs is sublinear. Therefore, one may ask what happens if graphs with large odd girth are considered. Many bounds for triangle-free graphs give somewhat better results when sharpened to graphs with large odd girth at least $2 k+1$, $k>1$. For instance, Theorem 5 can be improved by replacing $|\mathcal{C}|$ with $(2 k+1) t$, and also by sharpening Claim 3. However, in the latter we note that the parity of $k$ plays a role. For $k=1,2$, the constant $2 / 5$ is best possible, for $k=3,4,5 / 12$ is best possible, and so on. It seems an interesting question to determine if this is simply an anomaly of the method, or if the parity of $k$ affects the degree to which bounds on $i(G)$ can be strengthened.

\section{REFERENCES}

[1] A. Harutyunyan, P. Horn, and J. Verstraete. Independent dominating sets in graphs of girth five. submitted, 2009.

[2] J. Haviland. Independent domination in triangle-free graphs. Discrete Math., 308(16):3545-3550, 2008.

[3] J. Haviland. A note on independent domination in graphs of girth 5. Australas. J. Combin., 40:301-304, 2008.

[4] J. Haviland. A note on independent domination in graphs of girth 6. Australas. J. Combin., 42:171-175, 2008.

[5] J. H. Kim. The Ramsey number $R(3, t)$ has order of magnitude $t^{2} / \log t$. Random Structures Algorithms, 7(3):173207, 1995.

[6] C. Wang. The independent domination number of maximal triangle-free graphs. Australas. J. Combin., 42:129136,2008 .

(Goddard) School of Computing and Department of Mathematical Sciences, Clemson University, Clemson, SC USA, GOdDARD@CLEMSON.EDU

(Lyle) Department of Mathematics, The University of Southern Mississippi, Hattiesburg, MS USA, SAMUEL.LYLE@USM.EDU 